\title{
Advancement in Tritium Transport Simulations for Solid Breeding Blanket System
}

\author{
Alice Ying ${ }^{\mathrm{a}}$, Hongjie Zhang ${ }^{\mathrm{a}}$, Brad J Merrill ${ }^{\mathrm{b}}$, Mu-Young Ahn ${ }^{\mathrm{c}}$ \\ ${ }^{a}$ Mechanical and Aerospace Engineering Dept., UCLA, Los Angeles, CA 90095, USA \\ ${ }^{b}$ Idaho National Laboratory, Idaho Falls, ID 83415, USA \\ ${ }^{c}$ National Fusion Research Institute, Daejeon, Republic of Korea
}

In this paper, advancement on tritium transport simulations was demonstrated for a solid breeder blanket HCCR TBS, where multi-physics and detailed engineering descriptions are considered using a commercial simulation code. The physics involved includes compressible purge gas fluid flow, heat transfer, chemical reaction, isotope swamping effect, and tritium isotopes mass transport. The strategy adopted here is to develop numerical procedures and techniques that allow critical details of material, geometric and operational heterogeneity in a most complete engineering description of the TBS being incorporated into the simulation. Our application focuses on the transient assessment in view of ITER being pulsed operations. An immediate advantage is a more realistic predictive and design analysis tool accounting pulsed operations induced temperature variations which impact helium purge gas flow as well as $\mathrm{Q}_{2}$ composition concentration time and space evolutions in the breeding regions. This affords a more accurate prediction of tritium permeation into the He coolant due by accounting correct temperature and partial pressure effects and realistic diffusion paths. The analysis also shows that by introducing by-pass line to accommodate ITER pulsed operations in the TES loop allows tritium extraction design being more cost effective.

\section{Keywords: Numerical Modeling, Tritium Transport and Permeation, Purge Gas Flow, Ceramic Breeder Blankets}

\section{Introduction and Objectives}

Factors contributing to accuracy and fidelity of tritium transport predictions are: 1. Theoretical and phenomenological modeling of transport behavior; 2 . Material interface physics modeling and numerical treatment; 3. Verified codes with transport mathematical formulations; 4. Accurate material databases; 5. Inclusion of important geometric design features; and 6. Accounting for other physical phenomena such as temperature and fluid field in the analysis. Phenomenological tritium transport models and computational codes for blankets/FW have been developed through diverse effortsover the past several decades [for example 1-4]. However, confidence in accurate predictions of tritium transport from the codes remains low. Lack of confidence is due in part to scatter in material databases, but more importantly the models lacked integrated analysis where important physics such as temperature are incorporated.

In view of the increasing availability of verified multi-physics simulation tools in conjunction with advances in affordable high-performance computing, more complex and realistic tritium transport modeling is possible. With integrated models of fluid flow, energy, and tritium mass transport, observations of interesting tritium transport phenomena and detailed interactions between tritium mass and flow and temperature have been performed and observed [5-7]. However, these analyses were performed for a unit cell or in a 2-D domain, where still some important real-world phenomena such as multiple channel flow distribution as well as simulation of diffusion length in a 2-D model for 3-D channels were not properly represented.
Consequently, a modeling predictive capabilitythat simultaneously addresses the diverse, multi-physics and accounts for all materials, configurations, and heterogeneity details is still missing.

In this paper, we present an efficient numerical technique where detailed computational mesh decomposition for faithful reproduction of the transport phenomena can be achieved with affordable, off-theshelf computing power. The goal of tritium transport modeling is to achieve accurate tritium transport properties in the key areas of inventory, permeation, and extraction, using a HCCR TBS as a test bed. The need for more accurate 3-D simulations becomes more urgent as ITER TBM programs progress, in order to avoid excessively designed components and capacities. The analyses include estimating tritium concentrations in the He coolant lines, tritium concentration and form in the purge gas line, tritium leakage through piping, and tritium extraction efficiency. Our application specifically focuses on the transient assessments to match pulsed operations of ITER. The analyses will be particularly useful to project and help interpret experimental results of ITER, while simultaneously assisting TBM and tritium extraction system (TES) design and system layout in order to minimize consumptions of $\mathrm{H}_{2}$ and power.

In the HCCR TBM design, the main coolant flow in the breeding zone is in the toroidal direction, while the helium purge flow is in the poloidal direction (Figure 1). There are three breeder layers, three Be layers, and one graphite layer comprising a single breeding zone unit.Layers are separated by $10 \mathrm{~mm}$ thick, $670 \mathrm{~mm}$ long

author's email: ying@fusion.ucla.edu 
cooling plates,containing34 coolant channels of $7 \mathrm{~mm}$ diameter.

Purple: Breeder/Blue: Be/Green: Graphite
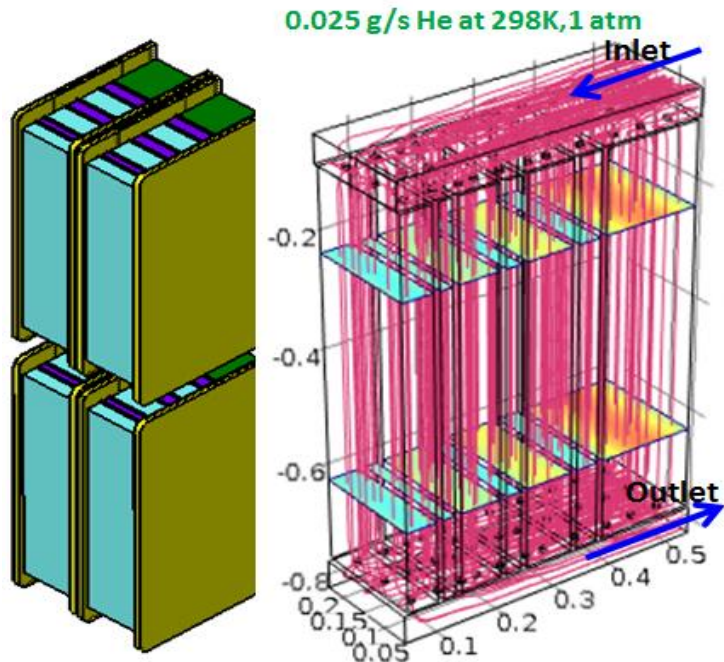

Fig. 1 Schematics of HCCR submodule breeding zone (left shown for four submodules) and one submodule breeding zone purge gas computational model (right)

\section{Computational Technique and Procedure}

The strategy adopted in this research is to leverage the advances of validated and verified open-source and commercial codes that satisfy the aforementioned requirements for accuracy in tritium transport modeling, thereby focusing on analysis and correct model setup over code development.

Considerable effort of this study has been in the development of a scheme to decompose the complete unit of a blanket module, and concomitant large computational domain involved in calculating tritium transport properties, into efficiently-handled smaller units. The major computational sub-domains proposed here are a helium purge breeding zone domain and a structure and cooling plate domain. Furthermore, the analysis is carried out in two steps. The 3-D helium purge gas velocity profiles and temperatures in the breeding zones are calculated in the first step. The results are passed into a tritium transport model; tritium concentrations in breeder, Be, graphite, and purge gas as well as in structural plates are calculated in the second step. This sequential step analysis is acceptable since there is only a one-way coupling between the first and second steps. Tritium transport properties will neither affect purge gas velocity nor temperature characteristics.

As illustrated in Figure 2, an "extrusion" technique of enforcing continuity of heat flux (from step 1) and hydrogen atomic flux is applied at the structure/purge gas interface. The extrusion technique involves data interpolation and data mapping to ensure the most appropriate nodal value is being used in the analysis. The use of two domains allows application of fine meshes when necessary, without burdening the simulation time of the entire model. For example, the pitch between the coolant channels in the cooling pate is $20 \mathrm{~mm}$. In order to capture the permeation to the coolant, the mesh along the poloidal direction in the cooling plate is made finer enough to provide 10 meshes in $20 \mathrm{~mm}$ span (Figure 3). In contrast, there is no such a requirement in the breeding zone, which has meshes in poloidal direction that are much larger. The total elements for the purge gas domain and structure domain are 103,612 and 359,304 , respectively.

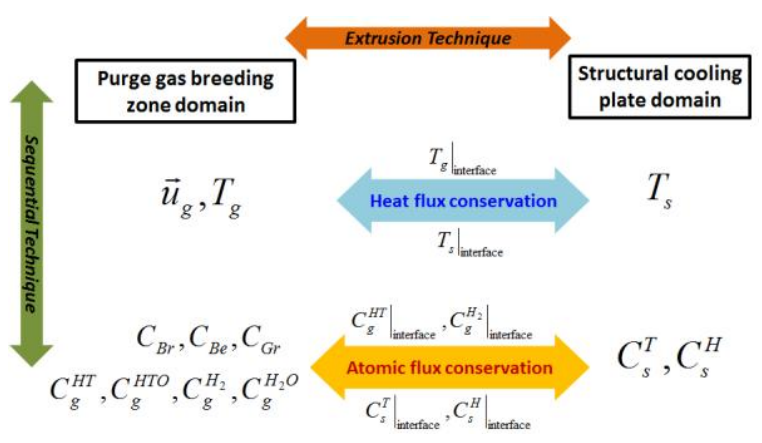

Fig. 2 Numerical procedure and technique for HCCR tritium transport analysis


Fig. 3 Meshes in purge gas breeding zone domain (top), and in structure and cooling plate domain (bottom) Insert shows the difference between purge and structure domain meshes

One of the challenges to achieve an accurate estimation of the tritium permeation into the He coolant involves reproducing tritium permeation path which varies along the cooling plate. However, meshing a rectangular plate with embedded circular coolant channels where a finer mesh size is needed near coolant channel boundariesconsumes significant computational memory. A 2-D tritium permeation study was conducted to calculate tritium permeative fluxes as well as inventory for a number of square channel sizes and for 
the actual cooling plate design. The idea here is to identify a square channel equivalent to circular channel with regard to tritium transport properties. The embracing of square channel allows using a sweep mesh technique with fewer meshes while achieving adequate accuracy with finer meshes in critical locations. The results shown in Figure 4 illustrate a square channel size of $5.8 \mathrm{~mm}$ used as an equivalent replacement to the actual circular channel design. The equivalent square channel used in the analysis is very good for reproducing the integrated permeative fluxes to the He coolant and to the Be region, and inventory; however, when a slightly reduced mapped mesh is used these properties can be reproduced better with a coolant channel size of $5.9 \mathrm{~mm}$. Figure 5 illustrates the differences in the flux and concentration profiles between the actual circular coolant and the equivalent square channel designs. The integrated permeative flux to the coolant for 34 cooling channels as in the cooling plate design is about $0.4 \%$ higher. On the other hand, the structural mapped mesh has substantially fewerissueswith numerical convergence.

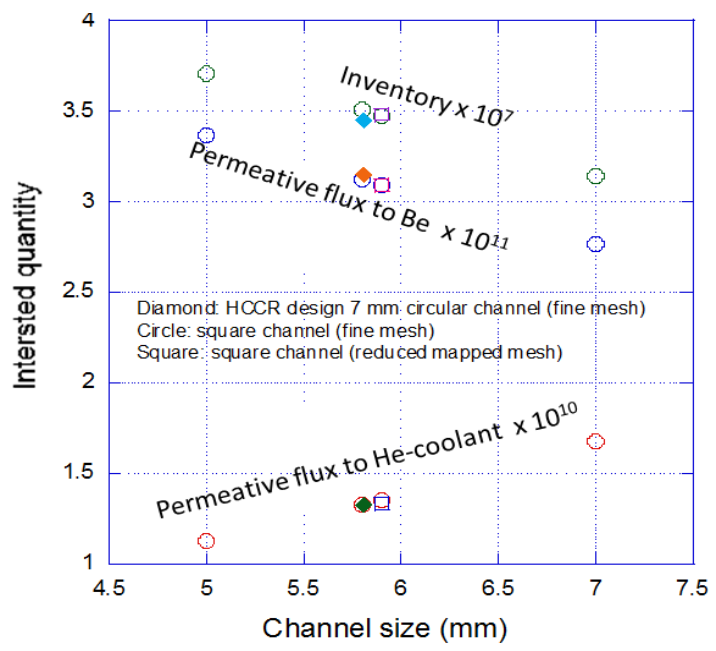

Fig.4 Tritium transport properties for different channel sizes and shapes

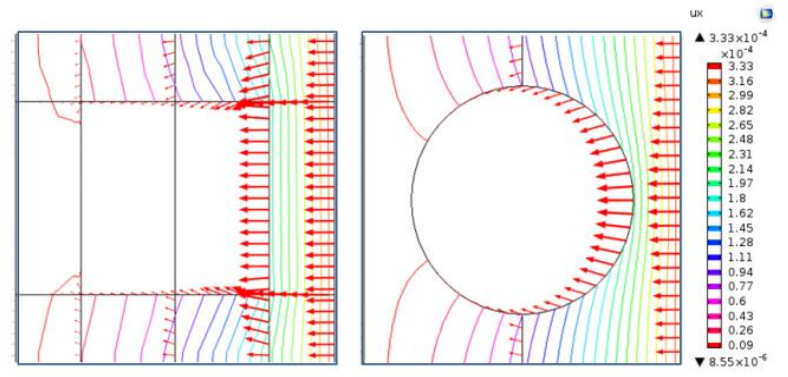

Fig. 5 Tritium concentration contour line and flux in the cooling plate (one channel shown): right: actual $7 \mathrm{~mm}$ diameter cooling channel design, left: equilibrium $5.9 \mathrm{~mm}$ square channel with reduced mapped mesh size

\section{Purge gas breeding zone model}

He purge gas velocity governs the hydrogen isotope $\mathrm{Q}_{2}$ partial pressure which in turnaffectsthe amount of tritium permeation into the cooling plates, and the design and operational requirements of TES (tritium extraction system). He purge gas velocity is also affected by temperature-dependent density.In this calculation, $0.025 \mathrm{~g} / \mathrm{s}$ of He purge gas enters the submodule at $298 \mathrm{~K}$ and $1 \mathrm{~atm}$. Although He purgeis not meant to remove the heat, nevertheless, its temperature increases poloidally due to amixing with the breeder/Be or graphite bulk temperature. However, in this model, conjugate heat transferand thermofluid modeling of the He coolant in the cooling plate was not included; instead a convective heat transfer boundary condition is applied at the coolant/structure boundary. The heat transfer coefficient as well as bulk temperature are estimated from analytical calculations, since this type of analysis has been proven to be feasible [5] thus no particular effort is devoted to this here.

The helium purge gas velocity in the breeding zone at different downstream locations and different cycles/times varies most during the $1^{\text {st }}$ cycle when the submodule is operated from a 'cold start' (room temperature after long plasma shutdown). As shown in Figure 6,the purge gas velocity has an M-shaped profile due to a higher void fraction near the wall. However, at the $3^{\text {rd }}$ cycle, the purge gas velocity becomes less sensitive to the downstream locationsince the temperature and therefore the density of the purge gas do not vary much. This is due to breeding zones, for all cycles after the second,beginning froma 'warm start' (the temperaturesof subsequent cycles approach the He coolant inlet temperature of $573 \mathrm{~K}$, see Figure 8). The average advection velocity in the breeding zone is approximately $1.3 \mathrm{~cm} / \mathrm{s}$.
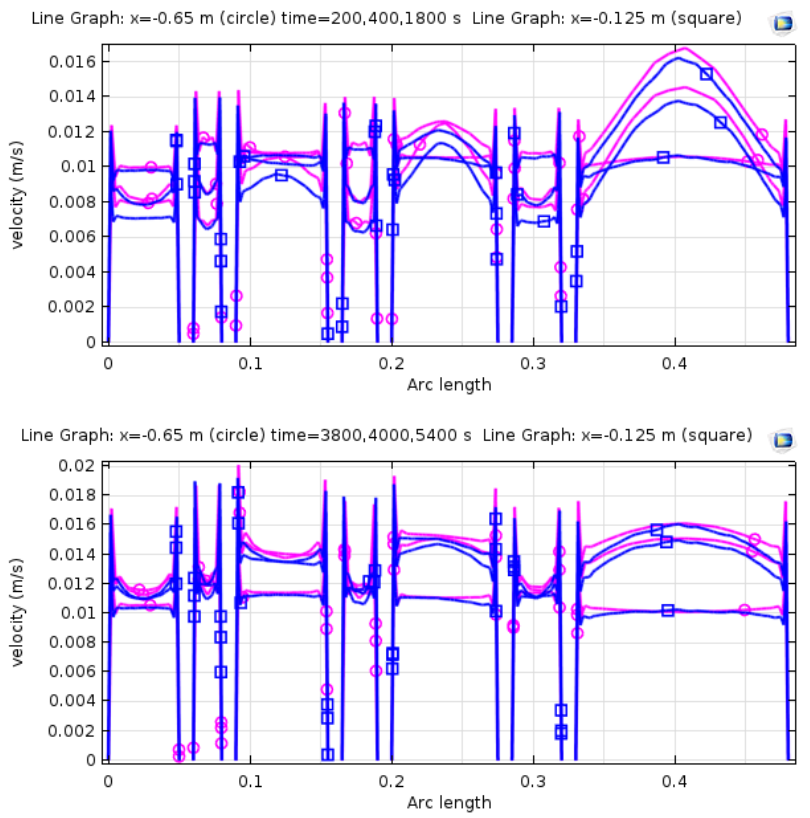

Fig.6 Velocity profiles at two downstream locations and at different times (Top- ${ }^{\text {st }}$ cycle from cold start; bottom: $3^{\text {rd }}$ cycle)

The purge gas average outlet temperature and velocity as a function of time are illustrated in Figure 8, where temperature reaches about $753 \mathrm{~K}$ at the peak of the burn. The figure also indicates that changes in both purge gas temperature and velocity are small after the second cycle. These calculated purge gas velocity and temperature profiles are fed into 3-D mass transport model for tritium partial pressure and transport 
properties assessment. This will be discussed in section 4.

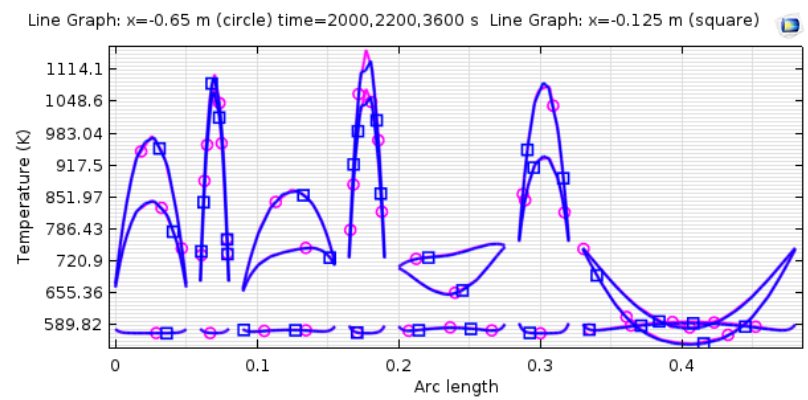

Fig. 7 Temperature profiles in various breeding zones at two downstream locations during $2^{\text {nd }}$ cycle of ITER inductive operation (after cold start)

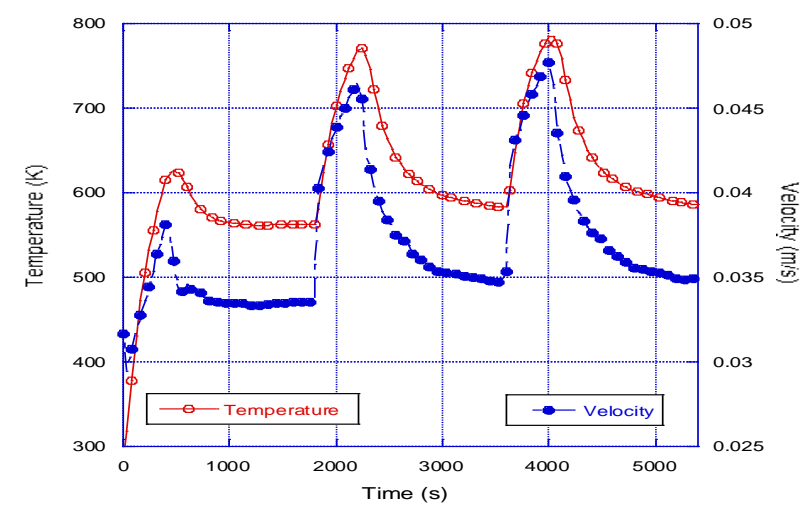

Fig.8 Purge gas outlet temperature and velocity indicating one cycle is adequate to reach equilibrium from cold start

\section{3-D Mass transport Analysis}

The principal chemical form for tritium in the purge gas stream was reported as HT because of the isotope exchange reaction between $\mathrm{T}_{2}$ and $\mathrm{H}_{2}$ [4]. However, due to the release of oxygen during lithium-neutron reaction, the knowledge of the concentration amounts of $\mathrm{H}_{2} \mathrm{O}$ and HTO in the purge gas is important and needed for performing the adsorption and desorption design analysis of a RTMS (room temperature molecular sieve). The hydrogen isotope mass balance equations as implemented in COMSOL [8] havethe general form:

$\frac{\partial C_{i}}{\partial t}+\nabla \cdot\left(-D_{i} \nabla C_{i}\right)+u \cdot \nabla C_{i}=R_{i}$

Where $i=\mathrm{HT}$, HTO, $\mathrm{H}_{2}, \mathrm{H}_{2} \mathrm{O}$; and $\mathrm{R}_{\mathrm{i}}$ is the tritium generation rate and/or isotopic exchange rate. The term $\mathrm{R}_{\mathrm{i}}$ is modified to include a term $\left(-\mathrm{C}_{\mathrm{i}} \cdot \nabla \mathrm{u}\right)$ to ensure concentration conservations due to purge gas velocity changes.

Tritium release from ceramic breeders is based on a residence time expression; while tritium release from $\mathrm{Be}$ is estimated by fractional release rate [4]. In this analysis, we assume a mixture of $50 \%$ HTO and $50 \%$ HT released to the He purge gas stream; based on the assumption one oxygen is released when two $\mathrm{Li}$ atoms are consumed. The released HTO form can be reduced to HT due to reactions with $\mathrm{H}_{2}$ through the isotope exchange reaction between tritium containing chemicals and hydrogen containing chemicals as:
$\mathrm{H}_{2}+\mathrm{HTO} \leftrightarrow \mathrm{HT}+\mathrm{H}_{2} \mathrm{O}$

Chemical equilibrium of the above reaction can be written as:

$K_{e q}(T)^{-1}=\frac{\left[H_{2}\right][H T O]}{[H T]\left[H_{2} O\right]}$

where the equilibrium constant is expressed by [9] as:

$\log _{10} K_{e q}(T)=0.292 \log _{10}(T)+(336.5 / T)-1.055$ [3]

COMSOL has built-in reaction solution functions to evaluate conversion rate and equilibrium concentrations based on the equilibrium constant of Eq. [3].

The concentrations of $\mathrm{H}$ and $\mathrm{T}$ in the reduced activation ferritic steel (RAFS) cooling plate just below the gas-RAFS interface in equilibrium with $\mathrm{P}_{\mathrm{H} 2}$, and $\mathrm{P}_{\mathrm{HT}}$ are expressed by modified Hickman's theory as [10]:

$$
\begin{aligned}
& C_{H}^{e q}=K_{s, H} P_{H_{2}} / P_{\text {total }}^{1 / 2}+1 / 2 K_{S, H T} P_{H T} / P_{\text {total }}^{1 / 2}[4] \\
& C_{T}^{e q}=1 / 2 K_{S, H T} P_{H T} / P_{\text {total }}^{1 / 2}[5] \\
& P_{\text {total }}=P_{H_{2}}+P_{H T}[6]
\end{aligned}
$$

Where $K_{s, H T}$, and $K_{s, H}$ are the Sieverts' constants for HT, and $\mathrm{H}_{2}$. Although it is desired to use $K_{s, H T}, K_{s, H}$ is used for both $\mathrm{H}_{2}$ and HT. Similarly to previous analysis [7], a recombination boundary condition is being used at the structure/coolant interface.

Figure 9 shows HT concentration profiles in various regions at 4000s of ITER operations (after cold start). Note that the results presented here are based on a halfsubmodule analysis. The $\mathrm{T}$ concentrations along the cooling plates are shown in Figure 10. $\mathrm{T}$ concentration increases along the poloidal plane. $\mathrm{T}$ concentration is lower next to the coolant channel. The HT concentrations along the breeding zone at different downstream locations are shown in Figure 11. The HT concentration increases as the purge gas moves downstream; however, its variations incorporate several factors including tritium generation, M-shaped velocity and equilibrium constant changes due to temperature changes. The $\mathrm{H}_{2}, \mathrm{HT}$, HTO and $\mathrm{H}_{2} \mathrm{O}$ concentrations at the purge gas outlet as a function of time are plotted in Figure 12. Some of these results are not intuitive until revealed through 3-D integrated multi-physics simulations. For example, $\mathrm{H}_{2}$ concentration drops much more during the burn primarily due to velocity increasing and slightly due to chemical interactions with $\mathrm{T}$ and $\mathrm{O}$ (since the amount of $\mathrm{T}$ atoms is significantly smaller the $\mathrm{H}$ atoms.). According to the analysis, the main tritium component in the He purge is HT, of which concentration in the purge gas outlet is about 40 times higher than HTO. The total (over the coolant surface areas) permeation ratesto the He coolant as a function of time compared with different analyzed cases are shown in Figure 13. The estimated total permeation rate with3$\mathrm{D}$ calculations is about 1.3 times higher than that of previous 2-D velocity corrected calculations[7], where only average temperatures were used for plasma on and off with no detailed evolution considered. Theestimation 
using the average plasma on- and off temperatures in 3$\mathrm{D}$ analysis is slightly lower than that using a detailed temperature evolution analysis. Thisimplies the importance of inclusion of the geometric details in the permeation calculations.

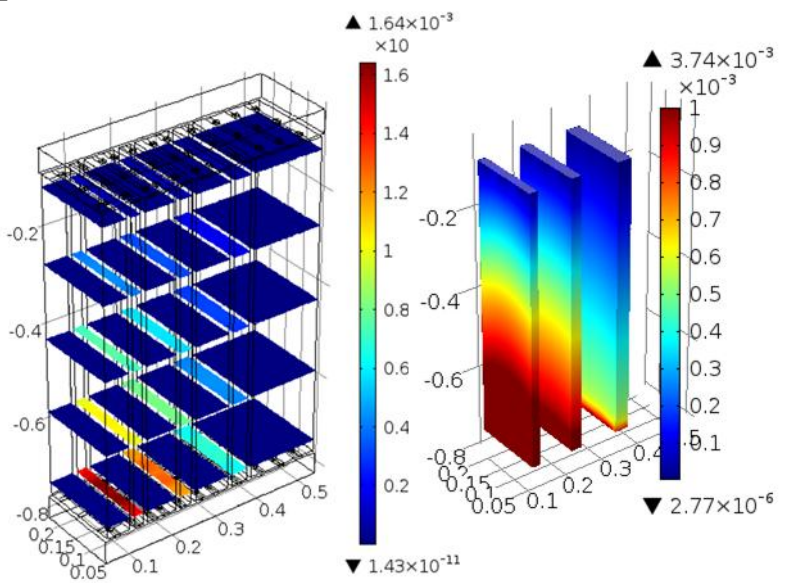

Fig. 9 Example HT concentrationprofiles in breeding zone (left) and three breeder layers

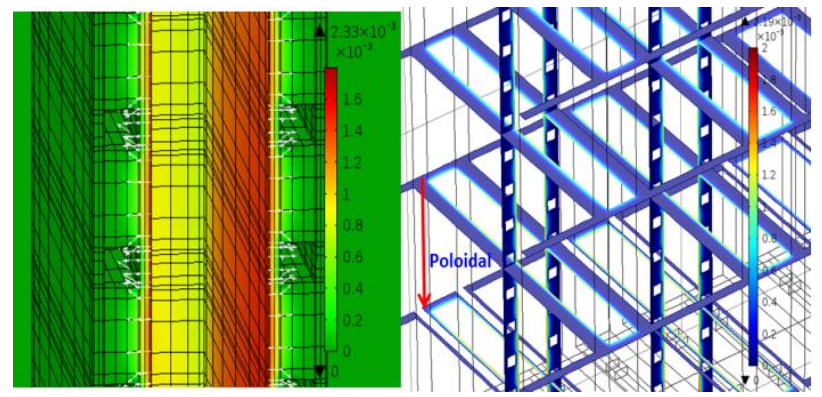

Fig.10 T concentration in cooling structure plate increases as He proceeds downstream. T concentrations also vary in the toroidal plane and cooling plate.

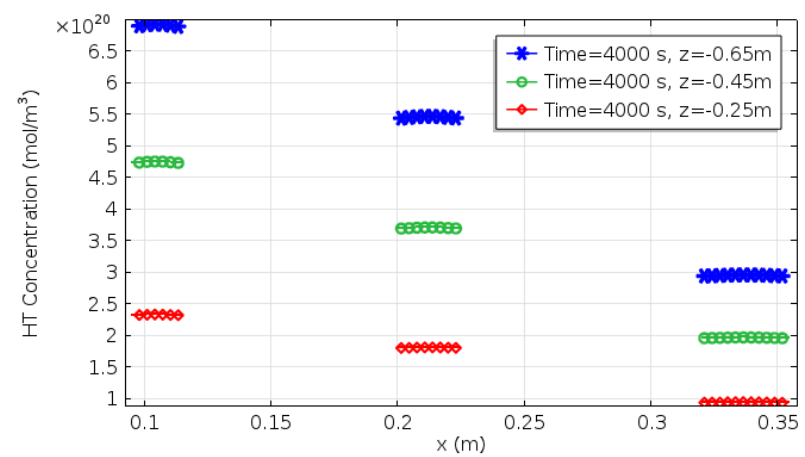

Fig.11 HT concentrations at various breeding layers at different poloidal planes at $4000 \mathrm{~s}$

\section{ITER Pulsed operation and TES design}

One of the design objectives as far as the tritium extraction fuel cycle is concerned is to minimize $\mathrm{H}_{2}, \mathrm{He}$, and power consumption. Design of tritium extraction system (TES) components such as CMSB (cryogenic molecular sieve bed) can be optimized for ITER's pulsed operational scenario to reduce $\mathrm{H}_{2}$ and powerconsumptions by incorporating a function to bypass TESduring plasma-off. As presented in the previous

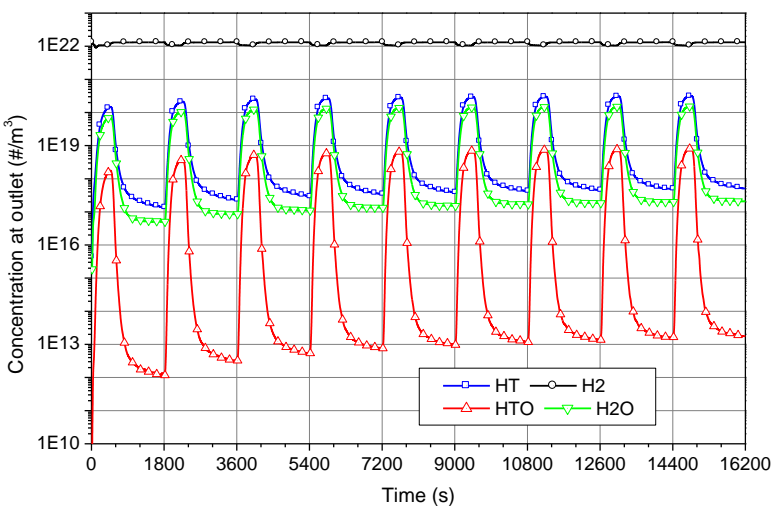

Fig. 12 Concentrations of $\mathrm{H}_{2}, \mathrm{HT}$, HTO, and $\mathrm{H}_{2} \mathrm{O}$ time revolution at the purge gas outlet

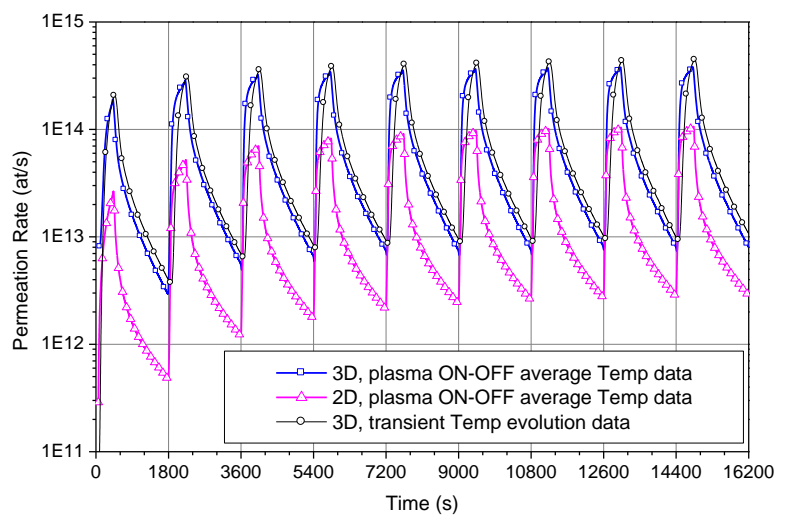

Fig. $13 \mathrm{~T}$ permeation rates into the He coolant as a function of time in one HCCR submodule for different simulations

section (Fig. 12), HT concentration drops as plasmashuts down. In the case where He purge streams, with its composition of $\mathrm{HT}$ and $\mathrm{H}_{2}$, are continuously processed through CMSB for removal of $\mathrm{HT}$ and $\mathrm{H}_{2}$, ignorant of the current plasma operational modes, analysis shows that the HT reaches its breakthrough at a much later time of around 6.1 days than that of $\mathrm{H}_{2}$ of around 3.3 days (see Figure 14). This implies that the $\mathrm{H}_{2}$ has to be made up during the first 3.3 days even though no tritium is being generated, while power has to be consumed toboth cool the He purge gas stream from outlet- to cryogenic temperatures and then heat fromcryogenic-back toinlet temperatures. The calculated CMSB uses 15 liters of MS5A (with an inner diameter of $0.31 \mathrm{~m}$ and a height of $0.2 \mathrm{~m}$ as an example CMSB design).

Since the retained tritium is only released from breeding materials until about 200s after the plasma is off, or the HT concentration in the purge gas reaches < $1 \%$ of its value during the burn. We propose inserting a by-pass line to be placed on-line at about $200 \mathrm{~s}$ after plasma shutdown. While operating with the by-pass line, the CMSB is seen to more effectively adsorb HT and reaches its saturated values at a higher value when $\mathrm{H}_{2}$ saturates at about 6 days as shown in Figure 14. Thus, the adsorption capacity of MS5A in CMSB is better utilized in operation with a plasma-off by-pass compared to the case of continuous operation. In addition, the adsorption capacity can increase by reducing the diameter of the column, which results in a higher purge gas velocity and a better mass transfer for the adsorption 
process. The concentration of the HT adsorbate in MS5A saturates at a higher value. For all cases analyzed, the inlet $\mathrm{HT}$ and $\mathrm{H}_{2}$ partial pressures are 1.6 $\mathrm{Pa}$ and $98.4 \mathrm{~Pa}$ during plasma on with a He flow rate of $0.1 \mathrm{~g} / \mathrm{s}$. The analysis is based on the mathematical models presented in Ref. [11]; models are solved in the COMSOL platform [8]. The concentration of the adsorbate in the adsorbent is described by the Langmuir-type mixed adsorption model with two independent active adsorption sites, where the Laugmuir constants for $\mathrm{H}_{2}$ and HT are also taken from Ref. [11].

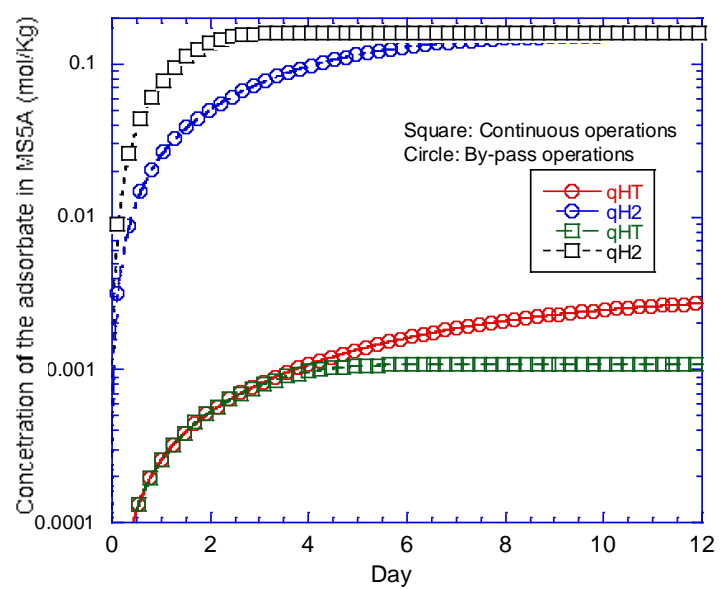

Fig.14 Adsorbed Q2 concentration evolution for continuous and by-pass operational scenarios

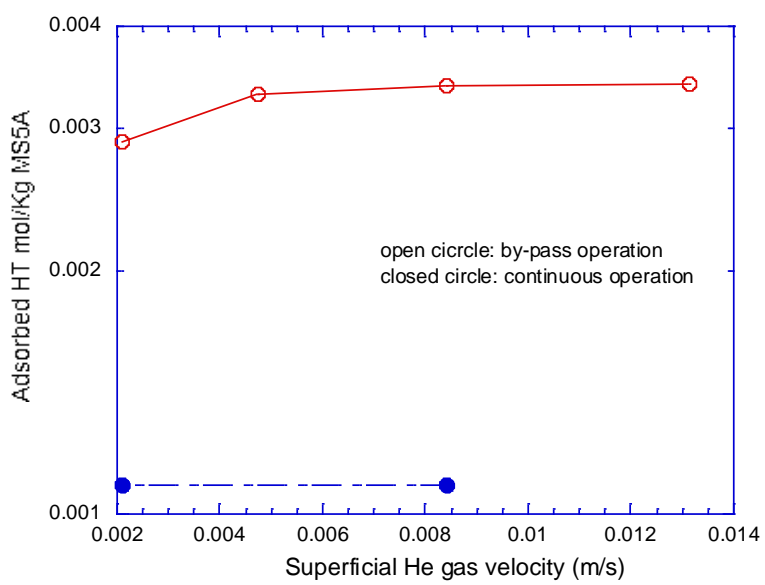

Fig. 15 Concentrations of the Q2 adsorbate in the adsorbent particle as a function of superficial velocity

\section{Significance and Discussions}

The paper presents a numerical procedure and technique where multi-physics simulations concerning various tritium transport properties evolutionsin a most complete engineering description of a blanket TBM can be simulated. The technique introduces an "extrusion" method to integrate two calculation geometric domains; while a sequential method is used in time evaluation to integrate two independent physics. The hydrogen isotope compositions in the purge gas stream as well as tritium permeation to $\mathrm{He}$ coolant through coolant-containing structures as a function of time are predicted. An immediate advantage is a predictive and design analysis tool for studying helium purge gas flow distribution among the breeding regions while reducing tritium permeation to the He coolant. The paper emphasizes transient analyses to track ITER pulsed operations, for whichan analysis is extended withbenefit to tritium extraction system design. The analysis shows that by introducing by-pass lines in TES loops allows tritium extraction in a more cost effective manner.

\section{ACKNOWLEDGMENTS}

This work was supported by the U. S. Department of Energy Contract DE-FG03-ER52123.CAD model and operational parameters of HCCR was supported by Task Agreement between UCLA-NFRI for Cooperation on R\&D for Fusion Nuclear Science to Expedite the Realization of Magnetic Fusion Energy.

\section{References}

[1] G. R. LONGHURST, D. F. HOLLAND, J. L. JONES, AND B. J. MERRILL, June, 1992, TMAP4 User'sManual, EGG-FSP-10315. TMAP7 User Manual, INEEL/EXT-0402352, October 2004.

[2] G. R. Longhurst, J. Ambrosek, Verification and Validation of the Tritium Transport Code TMAP7, Fusion Science and Technology, Vol. 48, No. 1,July 2005, Pages 468-471

[3] I. Ricapito, P. Calderoni, Y. Poitevin, L. Sedano, Tritium transport modeling for breeding blanket: tate of the art and strategy for future development in the EU fusion program, Fusion Engineering and Design 87 (2012) 793-797

[4] FabrizioFranza, Tritium transport analysis in HCPB DEMO blnaket with the FUS-TPC Code. KIT Scientific Report 7642, 2013

[5] A. Ying, J. Kotulski, H. Zhang, M. Ulrickson, M. Youssef, R. Munipalli, Virtual plasma chamber integrated multiphysics simulation: Status and next steps,Fusion Engineering and Design, Volume 89, Issues 7-8, October 2014, Pages 1144-1150

[6] H. Zhang, A. Ying, M. Abdou, Quantification of Dominating Factors in Tritium Permeation in PbLi Blankets, Fusion Science and Technology, Vol. 68, No. 2 , September 2015, dx.doi.org/10.13182/FST14-936

[7] Alice Ying, H. Zhang, M. Y. Anh, Y. Lee, Tritium transport evolutions in HCCR TBM under ITER Inductive Operations, Fusion Science and Technology, Vol. 68, No. 2 ,September 2015, dx.doi.org/10.13182/FST14-908

[8] COMSOL, v.5 2014. COMSOL, Inc., COMSOL Multiphysics User's Guide, Burlington, MA.

[9] Chang H. Oh, Eung S. Kim, September 2009, Development and Verification of Tritium Analyses Code for a Very High Temperature Reactor, INL/EXT-09-16743

[10] Satoru TANAKA \& Ryohei KIYOSE, Isotope Separation of Hydrogen and Deuterium by Permeation through Palladium Membrane, J. of Nuclear Science and Technology, 16[12], pp. 923-925 (December 1979).

[11] K.I. Tanaka, M. Uetake, M. Nishikawa, Calculation of Breakthrough Curve of Multicomponent Hydrogen Isotopes Using Cryosorption Column, J. of Nuclear Science and Technology, vol. 33, No.6, p. $492-503$ (June 1996) 